\title{
Unexpected lung lesions in high resolution computed tomography (HRTC) among patients with advanced HIV disease
}

\author{
S.A. Guillemi*, C.A. Staples**, J.C. Hogg', A.N. Le*++, L.M. Lawson*, \\ M.T. Schechter*++\#+, J.S.G. Montaner*+++
}

Unexpected lung lesions in high resolution computed tomography (HRTC) among patients with advanced HIV disease. S.A. Guillemi, C.A. Staples, J.C. Hogg, A.N. Le, L.M. Lawson, M.T. Schechter, J.S.G. Montaner. CERS Journals Ltd 1996.

ABSTRACT: The present pilot study was undertaken to characterize the frequency of lung lesions in asymptomatic human deficiency virus (HIV) infected individuals with advanced HIV disease.

Thirty two consecutive HIV + homosexual males assessed for initiation of Pneumocystis carinii pneumonia (PCP) prophylaxis, were prospectively studied. All patients underwent a complete medical history, physical examination, pulmonary function tests and high resolution computed tomography (HRCT). HRCT scans were read by a single radiologist, who was blind as to the clinical status of the patient.

Unexpected HRCT scan lesions were found in $60 \%$ of patients. There were no statistically significant differences between patients with normal and abnormal HRCT with respect to age, height, weight, CD4+ count, smoking history, serum albumin, alpha 1-antitrypsin level or body mass index. Forced vital capacity (FVC) (\% of predicted) and peak expiratory flow rate (PEFR) (\% pred) were not significantly different between groups. For patients with normal and abnormal HRCT forced expiratory volume in one second (FEV1) (\% pred) was $99 \pm 12$ vs 92 \pm 16, FEV1/FVC was 82 \pm 5 vs 76 \pm 9 , and forced mid-expiratory flow (FEF25-75) (\% pred) was $100 \pm 24$ vs $77 \pm 27$, respectively. There were no statistically significant differences between patients presenting with destructive versus nondestructive lung HRCT lesions.

Our results demonstrate that as many as $60 \%$ of $\mathrm{HIV}$-infected patients have unexpected abnormalities on HRCT at the time of starting PCP prophylaxis. We speculate that these lesions may contribute to the high frequency of spontaneous pneumothoraces previously reported in this patient population.

Eur Respir J., 1996, 9, 33-36.
*AIDS Research Programme, Dept of Medicine, ${ }^{* *}$ Dept of Radiology, and +Pulmonary Research Laboratory, St Paul's Hospital, University of British Columbia, Vancouver, BC, Canada. ${ }^{++}$Centre for Excellence in HIV and Related Diseases, Province of British Columbia, Vancouver, BC, Canada. \#Dept of Health Care and Epidemiology, Faculty of Medicine, University of British Columbia, Vancouver, BC, Canada. +British Columbia Centre for Excellence in HIV/AIDS, St Paul's Hospital, University of British Columbia, Vancouver, BC, Canada.

Correspondence: J.S.G. Montaner, AIDS Research Programme, St Paul's Hospital, University of British Columbia, 210-1033, Davie Street, Vancouver, B.C., Canada V6E 1M7

Keywords: Acquired immune deficiency syndrome, high resolution computed tomography, human immunodeficiency virus, pneumocystis carinii pneumonia

Received: March 71995

Accepted after revision August 161995

This work was supported in part by the National Health Research \& development Programme (NHRDP), Health and Welfare, Canada. J.S.G.M. is a National Health Research Scholar and M.T.S. is a National Health Research Scientist of the NHRDP.
A number of recent reports have identified unexpected lung abnormalities, including destructive lung lesions, among individuals with human immunodeficiency virus (HIV) disease [1-3]. In a limited number of cases, this has been described even prior to the initiation of Pneumocystis carinii pneumonia (PCP) prophylaxis or the development of acquired immune deficiency (AIDS)-defining conditions. In fact, a possible association between prolonged infection with HIV and the development of pulmonary emphysema has been described [1]. The high susceptibility of HIV-infected individuals to develop multiple pulmonary complications greatly complicates the study of this potential association. In addition, PCP prophylaxis is widely recommended among patients with a CD4+ count below 200 cells $\cdot \mathrm{mm}^{-3}$ [4]. Although current guidelines suggest that daily trimethoprim-sulphamethoxazole is the most effective regimen, intolerance is frequent in this patient population [5]. Intermittent aerosolized pentamidine, although less effective, is better tolerated [6, 7]. Recently, however, use of aerosolized pentamidine has been associated with an increased frequency of spontaneous pneumothoraces $[8,9]$. The underlying mechanism responsible for this association remains unclear. We hypothesized that an "emphysema-like pulmonary disease", as described by Diaz et al. [1] could predispose to the development of pneumothorax. Use of aerosolized pentamidine in turn, could worsen this process by virtue of its potentially heterogeneous distribution throughout the lung parenchyma (particularly among patients with underlying lung abnormalities) or through the promotion of cough, which is often associated with use of aerosolized pentamidine [7].

High resolution computed tomography (HRCT) of the lung has been repeatedly shown to be of value in the noninvasive assessment of lung lesions [10, 11]. This has 
been especially so in the context of destructive lung lesions, such as occurs among patients with emphysema. In fact, it has been suggested that HRCT is currently the most accurate method for diagnosing emphysema in vivo [12].

We therefore conducted the present study to assess the frequency of HRCT lung lesions among patients with advanced HIV disease referred for initiation of PCP prophylaxis, prior to the initiation of aerosolized pentamidine.

\section{Methods}

HIV-infected individuals referred for assessment regarding initiation of PCP prophylaxis were eligible for the study, with the exception of those with known or suspected active, respiratory disease. All patients underwent a complete medical history, physical examination, and blood tests, including complete blood count (CBC) and differential, haemoglobin $(\mathrm{Hb})$, mean corpuscular volume $(\mathrm{MCV})$, platelet count, liver function test, and absolute and fractional CD4+ and CD8+ cell counts. Pulmonary function tests (PFTs) (Sensor Medics PFT, 5), including forced vital capacity (FVC), forced expiratory volume in one second (FEV1), FEV1/FVC, forced-mid expiratory flow (FEF25-75), peak expiratory flow (PEFR), transfer factor of the lungs for carbon monoxide $(T \mathrm{~L}, \mathrm{CO})$, alveolar volume (VA), $T \mathrm{~L}, \mathrm{CO} / V \mathrm{~A}$, and total lung capacity (TLC) using helium dilution were measured. All the measurements were compared to normal values as reported by GOLDMAN and BECKLAKE [13]. HRTC scans were obtained using a General Electric 9800 scanner (GE Medical Systems, Milwaukee, WI, USA) with $1.5 \mathrm{~mm}$ collimation scans obtained at $1 \mathrm{~cm}$ intervals, from the lung apices to the bases. All laboratory and radiological evaluations were completed on an elective basis within 6 weeks. Alpha $_{1}$-antitrypsin levels were obtained in the last 11 patients studied.

HRCT scans were read at once by a single radiologist (CAS), who was not aware of the clinical status of the patients. The results of the HRCT scan were classified on the basis of a descriptive report provided by the radiologist to a second reviewer (SAG), who was also unaware of the clinical status of the patients. HRCT scans were classified into normal and abnormal, and the latter into those demonstrating parenchymal destruction (including apical cysts, paraseptal emphysema, emphysema, cyst or cavities), or parenchymal infiltration (including groundglass lesions, consolidation, nodules or linear opacities).

The results were analysed using t-test and analysis of variance unless otherwise indicated. All p-values were two-sided. All data are expressed as mean \pm standard deviation.

\section{Results}

A total of 32 consecutive patients were studied. All but one were homosexual males, and none reported prior recreational use of intravenous drugs. All had normal Chest radiographs at the time of initial evaluation. Unexpected HRCT scan lung lesions were found in 19 (60\%) patients.
Patients were divided in three groups according to the findings in the HRCT (fig. 1). Group I included 13 patients with normal HRCT; Group II included 10 patients with destructive parenchymal lesions; and Group III included 9 patients with parenchymal infiltrates. The parenchymal destruction identified included one or more of the following lesions in a given patient: apical cysts $(n=11)$, paraseptal emphysema $(n=8)$, emphysema $(n=8)$, isolated cysts or cavities $(n=3)$ and parenchymal infiltration included one or more of nodules $(n=11)$, ground-glass $(n=7)$, consolidation $(n=2)$ and multiple linear opacities $(n=1)$. Three patients had a prior history of PCP, all of whom had HRCT abnormalities, three had destructive changes (emphysema in two, cyst and cavities in one) all three also had some infiltrative changes (ground-glass in two, nodules in one).

a)

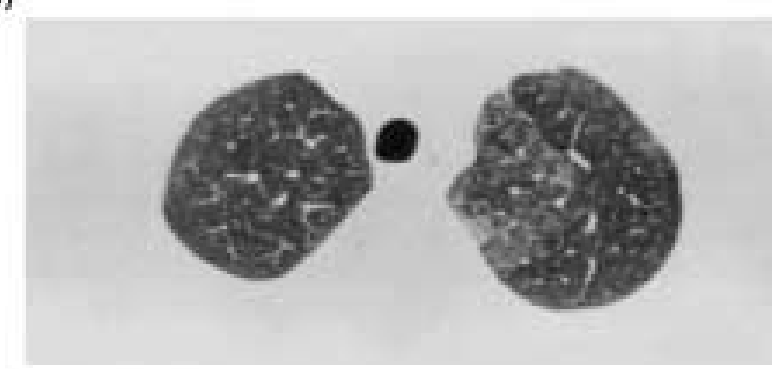

b)

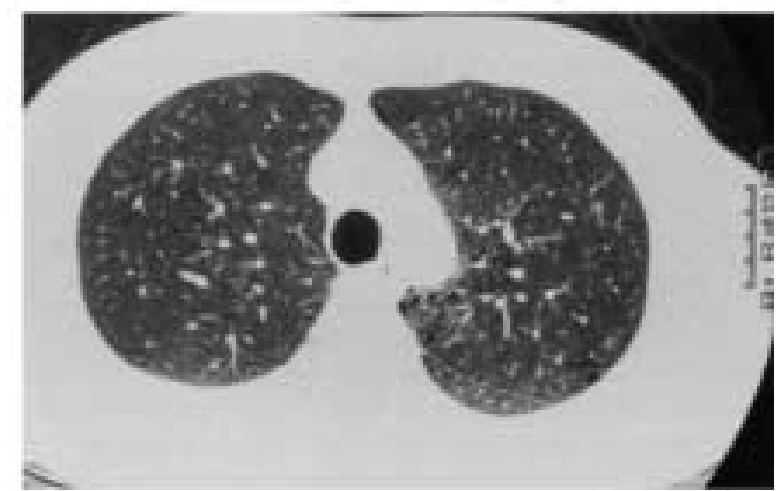

c)

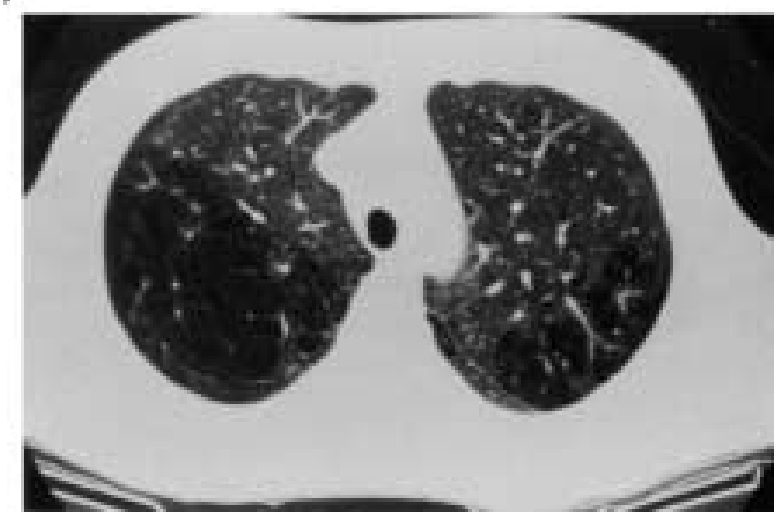

Fig. 1. - a) High resolution computed tomography (HRCT) demonstrating ground-glass opacities, predominantly in the left upper lung zone. b) HRCT demonstrating mild emphysema, predominantly in the area surrounding the aortic arch. c) HRCT demonstrating severe emphysema, predominantly in the right lung. 
Table 1. - Demographic laboratory and pulmonary function test data in patients with normal and abnormal findings on HRCT

\begin{tabular}{|c|c|c|c|c|}
\hline & $\begin{array}{c}\text { Pts } \\
\mathrm{n}\end{array}$ & $\begin{array}{c}\text { Normal } \\
n=13\end{array}$ & $\begin{array}{c}\text { Abnormal } \\
n-19\end{array}$ & p-value ${ }^{\dagger}$ \\
\hline Age yrs & 32 & $37 \pm 10$ & $39 \pm 7$ & 0.21 \\
\hline Height $\mathrm{cm}$ & 32 & $176 \pm 4$ & $176 \pm 6$ & 0.88 \\
\hline Weight $\mathrm{kg}$ & 32 & $72 \pm 9$ & $69 \pm 11$ & 0.49 \\
\hline $\mathrm{CD} 4+$ count cells $\cdot \mathrm{mm}^{-3}$ & 31 & $174 \pm 99$ & $192 \pm 153$ & 0.87 \\
\hline Smokers (ever) n & 25 & 8 & 17 & $0.09 *$ \\
\hline Smoking history pack/yrs & 25 & $18 \pm 12$ & $22 \pm 14$ & 0.37 \\
\hline Alpha $_{1}-\mathrm{AT} \mathrm{g} \cdot \mathrm{L}^{-1}$ & 11 & $178 \pm 32$ & $172 \pm 28$ & 0.71 \\
\hline Serum protein $\mathrm{g} \cdot \mathrm{L}^{-1}$ & 32 & $83 \pm 4$ & $76 \pm 7$ & 0.002 \\
\hline Serum albumin $\mathrm{g} \cdot \mathrm{L}^{-1}$ & 32 & $44 \pm 3$ & $41 \pm 6$ & 0.09 \\
\hline Body mass index & 32 & $23 \pm 3$ & $22 \pm 3$ & 0.48 \\
\hline FVC \% pred & 32 & $99 \pm 10$ & $99 \pm 14$ & 0.77 \\
\hline FEV1 \% pred & 32 & $99 \pm 12$ & $92 \pm 16$ & 0.06 \\
\hline FEV1/FVC & 32 & $82 \pm 5$ & $76 \pm 9$ & 0.03 \\
\hline FEF25-75 \% pred & 32 & $100 \pm 24$ & $77 \pm 27$ & 0.03 \\
\hline PEFR \% pred & 32 & $90 \pm 33$ & $95 \pm 25$ & 0.82 \\
\hline TL,CO \% pred & 31 & $92 \pm 13$ & $79 \pm 25$ & 0.103 \\
\hline$T \mathrm{~L}, \mathrm{CO} / V \mathrm{~A} \%$ & 31 & $93 \pm 14$ & $81 \pm 19$ & 0.061 \\
\hline TLC $\%$ pred & 31 & $98 \pm 7$ & $99 \pm 14$ & 0.76 \\
\hline
\end{tabular}

Data are presented as mean \pm SD. Alpha 1 -AT: alpha $_{1}$-antitrypsin; FVC: forced vital capacity; FEV1: forced expiratory volume in one second; FEF25-75: forced mid-expiratory flow; PEFR: peak expiratory flow rate: TL,CO: transfer factor of the lungs for carbon monoxide; VA: alveolar volume; TLC: total lung capacity; HRCT: high resolution computed tomography; \% pred: percentage of predicted value. ${ }^{\dagger}$ : t-test (two-tailed); *: Fisher's exact test (two-tailed).

As shown in table 1 , there were no statistically significant differences with regard to age, height, weight, smoking history, CD4+ count, serum albumin, alpha ${ }_{1}^{-}$ antitrypsin or body mass index between patients with normal or abnormal HRCT of the lung. Serum protein was statistically higher $(p=0.002)$ in patients with normal HRCT of the lung.

PFTs demonstrated no statistically significant difference with regard to FVC ( $\%$ of predicted), PEFR (\% pred), $T$ L,CO, $T$ L,CO/VA or TLC. FEV1, FEV1/FVC and FEF25-75 were statistically higher in patients with normal HRCT of the lung. Notably, this difference between groups was no longer present when the analysis was repeated excluding patients with prior history of $\mathrm{PCP}(\mathrm{n}=3)$, current pneumothorax $(n=1)$ and lung cancer $(n=1)$.

\section{Discussion}

A full $60 \%$ of the patients studied demonstrated unexpected lung lesions on HRCT scan. Among them, one half of the lesions where destructive in nature. Lung lesions ranged in severity from single lung cysts to varying degrees of emphysema; a lung cancer or a pneumothorax. In all instances, there was no clinical evidence of active lung disease on history or physical examination within 6 weeks prior to the HRCT scan.

We found no statistically significant differences with regard to demographic parameters between individuals who had normal or abnormal HRCT scans. It has previously been suggested that malnutrition and weight loss could be one of the links between emphysematous lung changes and advanced HIV infection [14]. In this context, it is of interest to note that a lower serum protein level was statistically associated with the presence of unexpected lung lesions in these patients. However, against this notion, our data showed that the body mass index and the serum albumin were not significantly different between groups and that the range of serum protein level was 78-91 and 66-90 $\mathrm{g} \cdot \mathrm{L}^{-1}$ in individuals with normal and abnormal HRCT scan, respectively, indicating considerable overlap. Together, these results would suggest that malnutrition and wasting are unlikely to explain the high frequency of unsuspected lung lesions in HRCT among our patients.

Alpha ${ }_{1}$-antitrypsin, measured in a limited number of patients, was not significantly different between groups. We cannot rule out, however, that differences may exist between patients with regard to the protease-antiprotease levels in the alveolar lining fluid. Whether this could contribute to the relatively high frequency of destructive lung lesions seen in our series remains to be addressed. Local inflammation or alveolitis either secondary to HIV or associated pathogens (i.e. P. carinii) could also contribute to the development of destructive lung lesions [15].

Our results also showed that patients with unexpected HRCT abnormalities had mild-to-moderate airflow limitation, with no significant change in TL,CO. Notably, this difference disappeared when three patients with previous PCP, one with a pneumothorax and one with a lung cancer were excluded from analysis. Furthermore, it must be stressed that the magnitude of the differences encountered were relatively small, and, therefore, would not allow the use of the PFTs as reliable clinical predictors of the presence of asymptomatic lung lesions on HRCT in the clinical setting.

A number of previous reports have described abnormal findings in HRCT of the lungs among a variety of immunosuppressed patients. KuHLMAN et al. [2] found evidence of bullous lesions in $42 \%$ of 55 patients with AIDS, (most of whom had a history of prior PCP). In the same study, only $16 \%$ of 50 neutropenic patients with acute leukaemia had bullous lesions in lung HRCT scans. SANDHU and GoODMAN et al. [16] reported a high incidence of pneumatoceles on chest radiograph in patients with PCP and AIDS, which resolved within 7 months. Finally, GURNEY and BATES [17] found lung cysts on conventional chest radiographs in AIDS patients, which were later shown to be scattered throughout the lungs on computed tomography. In contrast to our study, all of these reports focused on selected patients who had previously documented lung disease.

More recently, DiAz et al. [1] described a group of four young HIV-positive individuals presenting with dyspnoea, without a previous history of pneumonia or opportunistic infections. All four of them were found to have abnormal PFTs, with hyperinflation and a markedly decreased TL,CO. HRCT of the lungs revealed emphysema-like bullous changes in three of these patients. The authors concluded that there could be an association between prolonged HIV infection and the emphysemalike process, which could occur without a prior history of pulmonary infection. These four 
patients were among 32 consecutive HIV-positive patients undergoing complete PFT at their institution, 22 of which had recently recovered from PCP. Their PFT data suggest an interesting pattern of considerable air-trapping, hyperinflation and reduced transfer factor but little or no airway obstruction. This is an apparent contrast to our PFT results. However, it should be noted that our patient population was at an earlier stage with regards to their HIV disease and that only three patients in our series had a history of prior PCP.

Previous reports [6] have identified an association between the development of pneumothorax and use of aerosolized pentamidine for the purpose of PCP prophylaxis in patients with advanced HIV disease. A similar association between HRCT emphysema-like lesions and spontaneous pneumothoraces was recently described among nonsmoking individuals [18]. We speculate that unsuspected lung lesions, in particular those destructive in nature, may be partially accountable for this association. Further prospective studies will be required to confirm this hypothesis.

Our study does not offer any insight into the aetiology of the HRCT lung lesions found. Clinical followup of these patients allowed us to confirm a previously unrecognized lung cancer and a case of PCP. Of interest, the latter patient had developed a pneumothorax at the time of HRCT. All other patients remained free of overt lung disease for at least 3 months after the HRCT was performed.

In conclusion, our data suggest that there is a high prevalence of unsuspected lung lesions among individuals with advanced HIV disease even prior to initiation of PCP prophylaxis. We do not believe that our data should be interpreted as supporting routine HRCT screening of patients with advanced PCP. However, our data confirm that clinically and radiologically unrecognized destructive lung lesions of varying severity are relatively common in this group of patients. Furthermore, we speculate that destructive lung lesions, present in nearly one third of our study population, could be partially responsible for the recently described association between use of aerosolized pentamidine and spontaneous pneumothorax.

Acknowledgements: The authors are indebted to the study participants for their co-operation and to D. HamannTrou and K. Hsu for their superb secretarial support.

\section{References}

1. Diaz PT, Clanton TL Pacht ER. Emphysema-like pulmonary disease associated with human immunodeficiency virus infection. Ann Intern Med 1992; 116: 124-128.
2. Kuhlman JE, Knowles MC, Fishman EK, Siegelman SS. Premature bullous pulmonary damage in AIDS: CT diagnosis. Radiology 1989; 173: 23-26.

3. Panicek DM. Cystic pulmonary lesions in patients with AIDS. Radiology 1989; 173: 12-14.

4. Centers for Disease Control. Guidelines for prophylaxis against Pneumocystis carinii pneumonia for persons infected with human immunodeficiency virus. $M M W R$ 1989; 38: 1-9.

5. Hardy WD, Feinberg J, Finkelstein DM, et al. A controlled trial of trimethoprimsulfamethoxazole or aerosolized pentamidine for secondary prophylaxis of Pneumocystis carinii pneumonia in patients with the acquired immunodeficiency syndrome. AIDS Clinical Trial Group Protocol 021. N Engl J Med 1992; 327: 1842-1848.

6. Leong GS, Feigal DW, Montgomery AB, et al. Aerosolized pentamidine for prophylaxis against Pneumocystis carinii pneumonia. The San Francisco Community Prophylaxis Trial. $N$ Engl J Med 1990; 323: 769-775.

7. Montaner JSG, Lawson L, Gervais A, et al. Aerosol pentamidine in the secondary prophylaxis of AIDS-related Pneumocystis carinii pneumonia: a randomized placebocontrolled study. Ann Intern Med 1991; 114: 948-953.

8. Sepkowitz KA, Telzak EE, Gold JWM, et al. Pneumothorax in AIDS. Ann Intern Med 1991; 144: 455-459.

9. Newsome GS, Ward JD, Pierce PF. Spontaneous pneumothorax in patients with acquired immunodeficiency syndrome treated with prophylactic aerosolized pentamidine. Arch Intern Med 1990; 150: 2167-2168.

10. Morrison NJ, Abboud RT, Muller NL, et al. Comparison of single-breath carbon monoxide diffusing capacity and pressure-volume curve in detecting emphysema. Am Rev Respir Dis 1989; 141: 1179-1187.

11. Klein JS, Gamsu G, Webb WR, Golden JA, Muller NL. High resolution CT diagnosis of emphysema in symptomatic patients with normal chest radiographs and isolated low diffusing capacity. Radiology 1991; 182: 817-821.

12. Muller NL. CT diagnosis of emphysema. It may be accurate, but is it relevant? Chest 1993; 10: 329-330.

13. Goldman HI, Becklake MR. Normal values at median altitude and the prediction of normal results. Am Rev Tuberc 1959; 76: 457-467.

14. Sahebjani H. Emphysema-like changes in HIV. Ann Intern Med 1992; 116: 867.

15. Ramaswamy G, Jagadha V, Tchertkoff V. Diffuse alveolar damage and interstitial fibrosis in acquired immunodeficiency syndrome patients without concurrent pulmonary infection. Arch Pathol Lab Med 1985; 109: 408-412.

16. Sandhu JS, Goodman PC. Pulmonary cysts associated with Pneumocystis carinii pneumonia in patients with AIDS. Radiology 1989; 173: 33-35.

17. Gurney JW, Bates FT. Pulmonary cystic disease: comparison of Pneumocystis carinii pneumatoceles and bullous emphysema due to intravenous drug abuse. Radiology 1989; 173: 27-31.

18. Eklund G, Odont D, Hedenstierna G, Wiman LG. Nonsmoking, non-alpha ${ }_{1}$ antitrypsin deficiency-induced emphysema in nonsmokers with healed spontaneous pneumothorax, identified by computed tomography of the lungs. Chest 1991; 103: 433-438. 